This item was submitted to Loughborough's Research Repository by the author.

Items in Figshare are protected by copyright, with all rights reserved, unless otherwise indicated.

\title{
A Fubini theorem for pseudo-Riemannian geodesically equivalent metrics
}

\section{PLEASE CITE THE PUBLISHED VERSION}

http://dx.doi.org/10.1112/jlms/jdp032

\section{PUBLISHER}

Oxford Journals (@ London Mathematical Society)

\section{VERSION}

SMUR (Submitted Manuscript Under Review)

\section{PUBLISHER STATEMENT}

This work is made available according to the conditions of the Creative Commons Attribution-NonCommercialNoDerivatives 4.0 International (CC BY-NC-ND 4.0) licence. Full details of this licence are available at: https://creativecommons.org/licenses/by-nc-nd/4.0/

\section{LICENCE}

CC BY-NC-ND 4.0

\section{REPOSITORY RECORD}

Bolsinov, Alexey V., Volodymyr Kiosak, and Vladimir S. Matveev. 2019. "A Fubini Theorem for Pseudoriemannian Geodesically Equivalent Metrics". figshare. https://hdl.handle.net/2134/15932. 


\title{
A Fubini Theorem for pseudo-Riemannian geodesically equivalent metrics
}

\author{
Alexey V. Bolsinov, Volodymyr Kiosak and Vladimir S. Matveev
}

\begin{abstract}
We generalize the following classical result of Fubini to pseudo-Riemannian metrics: if three essentially different metrics on an $(n \geq 3)$-dimensional manifold $M$ share the same unparametrized geodesics, and two of them (say, $g$ and $\bar{g}$ ) are strictly nonproportional (i.e., the minimal polynomial of the $g$-self-adjoint $(1,1)$-tensor defined by $\bar{g}$ coincides with the characteristic polynomial) at least at one point, then they have constant sectional curvature.
\end{abstract} MSC: 53A20, 53B21, 53C22, 53C50, 53D25, 70G45, 70H06, 70H33, 58J60

\section{Introduction}

\subsection{Definitions and results}

Two Riemannian or pseudo-Riemannian metrics on the same manifold are said to be geodesically equivalent, if they have the same geodesics considered as unparametrized curves. Two metrics $g$ and $\bar{g}$ are strictly nonproportional at a point $x \in M$, if the minimal polynomial of the $g$-selfadjoint (1,1)-tensor $G$ defined by $\bar{g}$, i.e., $G_{j}^{i}:=g^{i k} \bar{g}_{k j}$, coincides with the characteristic polynomial of $G$. If one of the metrics is Riemannian, strict nonproportionality means that all eigenvalues of $G$ have multiplicity one.

The main result of the present paper is the following

Theorem 1. Let $g, \bar{g}$ and $\hat{g}$ be three geodesically equivalent metrics on a connected manifold $M^{n}$ of dimension $n \geq 3$. Suppose there exists a point at which $g$ and $\bar{g}$ are strictly nonproportional, and a point at which $g, \bar{g}$ and $\hat{g}$ are linearly independent. Then, the metrics $g, \bar{g}$ and $\hat{g}$ have constant sectional curvature.

If the metrics are Riemannian, the local version of Theorem 1 was proved by Fubini in [12, 13]. The proof of Fubini is short and elegant, but unfortunately, does not work in the pseudoRiemannian case. More precisely, Fubini's proof is based on the following classical result: 
Theorem 2 (Levi-Civita [20]). Assume $g$ and $\bar{g}$ are Riemannian geodesically equivalent metrics that are strictly nonproportional at a point $p$. Then, in a neighborhood of this point there exists a coordinate system $\left(x_{1}, \ldots, x_{n}\right)$ such that the metrics $g, \bar{g}$ are as follows:

$$
\begin{aligned}
& d s_{g}^{2}=\sum_{i=1}^{n}\left[\left|\prod_{\substack{j=1 \\
j \neq i}}^{n}\left(X_{i}\left(x_{i}\right)-X_{j}\left(x_{j}\right)\right)\right| d x_{i}^{2}\right], \\
& d s_{\bar{g}}^{2}=\sum_{i=1}^{n}\left[\frac{1}{X_{i}\left(x_{i}\right) \prod_{\alpha=1}^{n} X_{\alpha}\left(x_{\alpha}\right)}\left|\prod_{\substack{j=1 \\
j \neq i}}^{n}\left(X_{i}\left(x_{i}\right)-X_{j}\left(x_{j}\right)\right)\right| d x_{i}^{2}\right],
\end{aligned}
$$

where $X_{i}$ is a positive function of the variable $x_{i}$ only. Moreover, any two metrics of this form are geodesically equivalent.

Fubini calculated the Riemannian curvature $R_{i j, k m}$ of the metric (1) and observed that the component $R_{i j, k m}$ is non-zero only if $(i, j)=(k, m)$ or if $(i, j)=(m, k)$. Nowadays, such curvature tensors are called diagonal, see for example [53]. Then he showed that, unless the sectional curvature is constant, the curvature tensor determines the coordinates lines $\left(x_{1}, \ldots, x_{n}\right)$ uniquely. Thus, the metrics $g, \bar{g}$ and $\hat{g}$ must be simultaneously diagonalisable in some coordinate system and a short analysis shows that they are linearly dependent at every point.

Our proof, which is also valid in the pseudo-Riemannian setting, is as follows. We study the partial differential equation (5) which is responsible for the fact that the metric $\bar{g}$ is geodesically equivalent to $g$. The unknown functions in this equation are the components of a $(0,2)$-tensor $a_{i j}$ canonically constructed by the metric $\bar{g}$. Then, we find the integrability conditions for this equation. This is a system of linear equations on $a$ and the Hessian of $\lambda:=\frac{1}{2} a_{\alpha \beta} g^{\alpha \beta}$; the coefficients in these equations are algebraic expressions of entries of the curvature tensor and the metric. We show that if the system has two solutions, then for some $K \in \mathbb{R}$ the components of the curvature must satisfy the condition $a_{i}^{\alpha} Z_{\alpha j k l}+a_{j}^{\alpha} Z_{\alpha i k l}=0$, where $Z_{i j k l}=R_{i j, k l}-K \cdot\left(g_{i l} g_{j k}-g_{i k} g_{j l}\right)$. Then, it is an easy exercise in linear algebra to show that if in addition the metrics $g$ and $\bar{g}$ are strictly nonproportional, then this condition on the curvature implies that the curvature is actually constant. Then, the sectional curvature of the metrics $\bar{g}$ and $\hat{g}$ is constant as well by the Beltrami Theorem.

Remark 1. We emphasize that the essential part of our proof is, in fact, the analysis of algebraic properties of the integrability conditions for the "geodesic equivalence equation" (5), see Section 2. Algebraic aspects of the integrability conditions will also be clarified in Section 3 where we discuss an unexpected relationship between geodesically equivalent metrics and the so-called sectional operators on semisimple Lie algebras. As an application of this observation, we give an alternative, pure algebraic proof of the (local version of the) Fubini theorem, and we believe that this idea might be useful in a wider context. 
Remark 2. All assumptions in Theorem 1 are important. Indeed:

- if the dimension $n$ is 2, counterexamples were constructed in [16], see also [8];

- if the metrics are not strictly nonproportional, counterexamples can be found in $\S 9$ of [45], see also [43, 46, 47];

- if we omit the assumption that $g, \bar{g}$, and $\hat{g}$ are linearly independent, then we can take $\hat{g}=\bar{g}$, and the Levi-Civita metrics (1) and (2) for generic $X_{i}$ give a counterexample to the extension of Theorem 1.

\subsection{Motivation}

The first motivation, which was the reason why Fubini studied this question, came from the study of projective vector fields of pseudo-Riemannian metrics. Recall that a vector field $v$ is projective with respect to the metric $g$ if its (local) flow takes geodesics to geodesics. Projective and affine vector fields are very classical objects of investigation: both Lie [21] and Schouten [41] explicitly formulated the problem of constructing all metrics admitting one or sufficiently many projective vector fields. As a direct corollary of Theorem 1 we obtain

Corollary 1. Suppose that $g$ and $\bar{g}$ are geodesically equivalent metrics of a nonconstant sectional curvature on a connected manifold $M^{n}$ of dimension $n \geq 3$. Suppose there exists a point where $g$ and $\bar{g}$ are strictly nonproportional. Then, the dimension of the space of projective vector fields minus the dimension of the space of homothetic vector fields is at most one.

Recall that the vector field $v$ is homothetic, if $L_{v} g=$ const $\cdot g$, where $L_{v}$ is the Lie derivative. We allow the case const $=0$, so Killing vector fields are also homothetic.

Remark 3. In dimension 2, Corollary 1 does not hold. Indeed, Darboux-superintegrable metrics admit three projective vector fields and, as a rule, only one homothetic vector field, which is Killing. The definition of Darboux-superintegrable metrics and a description of their projective vector fields can be found in [8]. Moreover, it is possible to show that there are no other counterexamples to the 2-dimensional version of Corollary 1 . This is a very nontrivial statement which follows from the results of $[8,36]$, where all 2-dimensional metrics admitting projective vector fields were constructed.

Moreover, it is possible to use Theorem 1 in order to describe all projective vector fields of a metric $g$ under the assumptions that there exists a metric $\bar{g}$ which is geodesically equivalent to $g$ and strictly nonproportional to $g$. Indeed, in this case the system of PDEs on $v$ is a system of ODEs, which is much easier to analyse than a system of PDEs, see $[1,3,8,12,29,30,31,35,36]$, where this ODE-system was obtained and completely solved in particular cases. 
One more motivation comes from the theory of superintegrable systems. Recall that a metric is called superintegrable, if the number of independent integrals of special form is greater than the dimension of the manifold. There are different possibilities for the special form of integrals; de-facto the most standard special form of the integrals is that of the so-called Benenti-integrals, which are essentially the same as geodesically equivalent strictly nonproportional metrics, see [4, $7,17]$. The results of the present paper show that Benenti-superintegrable metrics of nonconstant curvature cannot exist, which was a folkloric conjecture.

\section{$1.3 \quad$ History}

The theory of projective transformations has a long and fascinating history. The first nontrivial examples of projective transformations were discovered by Lagrange [19], see also [33]. Geodesically equivalent metrics were studied by Beltrami, Darboux, Levi-Civita [20], Painlevé, Fubini [12, 13], Eisenhart, Weyl [52] and other classics. One can find more historical details in the surveys $[3,9,37]$ and in the introductions to the papers $[25,26,27,28,32,34,35,50]$.

The pseudo-Riemannian version of Fubini's Theorem was investigated by the Kazan school of geometry, in particular by A. Aminova and her collaborators. They tried, in particular, to mimic the Fubini proof for pseudo-Riemannian metrics by using the description of geodesically equivalent pseudo-Riemannian metrics obtained by Aminova [2], which is a generalization of Levi-Civita's Theorem 2. Unfortunately, the description by Aminova is very complicated, which makes this program very hard computationally. Besides, there are infinitely many different types of normal forms for pseudo-Riemannian metrics (depending on the Jordan form of the tensor $G$ ), and each of these types requires a separate investigation. According to [54], they proved Theorem 1 in dimensions up to 6 , though we did not find the place where the proof is written.

\section{Acknowledgements}

Alexey Bolsinov thanks Russian Foundation for Fundamental Research for partial financial support and Mathematisches Institut, Friedrich-Schiller-Universität Jena for hospitality. Vladimir Matveev is grateful to R. Bryant and M. Eastwood for useful discussions, and MSRI for the hospitality. Volodymyr Kiosak and Vladimir Matveev thank Deutsche Forschungsgemeinschaft (Priority Program 1154 - Global Differential Geometry) and FSU Jena for partial financial support. We are grateful to the referee and Ian Marshall for grammatical and stylistic corrections. 


\section{Proof}

\subsection{Schema of the proof}

We first show (Section 2.3) that if two geodesically equivalent metrics are strictly nonproportional at least at one point, then they are strictly nonproportional at almost every point. Then, we will show (Section 2.4) that if $g, \bar{g}$, and $\hat{g}$ are linearly dependent at every point of some neighborhood, they are linearly dependent at every point of the manifold.

Combining these two observation, we see that if the assumptions of Theorem 1 are fulfilled at one point, they are fulfilled at almost every point, i.e., though the theorem is global, it is sufficient to prove it locally. This will be done in Sections 2.5, 2.6.

\subsection{What we will use in the proof}

There are many tensor reformulations of the condition "the metrics $g$ and $\bar{g}$ are geodesically equivalent". In our paper we will use the following one, which was suggested by Sinjukov [44], see also $[7,10]$ : given two metrics $g$ and $\bar{g}$, consider the $(0,2)$-tensor

$$
a_{i j}:=\left|\frac{\operatorname{det}(\bar{g})}{\operatorname{det}(g)}\right|^{\frac{1}{n+1}} \cdot g_{i \alpha} \bar{g}^{\alpha \beta} g_{j \beta},
$$

and the function

$$
\lambda:=\frac{1}{2} a_{\alpha \beta} g^{\alpha \beta}
$$

where and $g^{\alpha \beta}$ and $\bar{g}^{\alpha \beta}$ denote the dual tensors to $g_{i j}$ and $\bar{g}_{i j}$ respectively, i.e., $g^{i \alpha} g_{\alpha j}=\delta_{j}^{i}$ and $\bar{g}^{i \alpha} \bar{g}_{\alpha j}=\delta_{j}^{i}$.

Theorem 3. [7, 10, 44] The metrics $g$ and $\bar{g}$ are geodesically equivalent, if and only if

$$
a_{i j, k}=\lambda_{, i} g_{j k}+\lambda_{, j} g_{i k}
$$

where the covariant derivative is taken with respect to the (Levi-Civita connection of the) metric $g$.

We will also use the following connection between geodesically equivalent metrics and integrable geodesic flows due to [24].

Let $a_{j}^{i}$ be as in (3), with one index lifted by $g$ (so now $a$ is a $(1,1)$-tensor, self-adjoint with respect to $g$ ). Consider the family $S_{t}$ of $(1,1)$-tensors

$$
S_{t} \stackrel{\text { def }}{=} \operatorname{det}(a-t \mathrm{Id})(a-t \mathrm{Id})^{-1}, \quad t \in \mathbb{R} \text {. }
$$


Remark 4. Although $(a-t \mathrm{Id})^{-1}$ is not defined for $t$ lying in the spectrum of $a$, the tensor $S_{t}$ is well-defined for every $t$. Actually, the matrix of $S_{t}$ is the comatrix of the matrix of $a-t \mathrm{Id}$. In particular, $S_{t}$ is a polynomial in $t$ of degree $n-1$ with coefficients being $(1,1)$-tensors.

We will identify the tangent and cotangent bundles of $M^{n}$ by $g$. This identification allows us to transfer the natural Poisson structure from $T^{*} M^{n}$ to $T M^{n}$.

Theorem $4([24,49,48,50,28])$. If a is a solution of $(5)$, then for every $t \in \mathbb{R}$ the function

$$
I_{t}: T M^{n} \rightarrow \mathbb{R}, \quad I_{t}(\xi) \stackrel{\text { def }}{=} g\left(S_{t}(\xi), \xi\right)
$$

is an integral for the geodesic flow of $g$.

Recall that a function is an integral of the geodesic flow, if it is constant along the trajectories, i.e., along the curves on the tangent bundle of the form $(\gamma(t), \dot{\gamma}(t))$, where $\gamma$ is a geodesic and $\dot{\gamma}(t)$ is its velocity vector at a point $t$.

We will also use the following statement, whose Riemannian version was obtained in [24], and pseudo-Riemannian generalisation is due to Topalov [48]. As in the introduction, we denote by $G$ the $(1,1)$-tensor $G_{j}^{i}:=g^{i k} \bar{g}_{k j}$.

Theorem 5 (follows from Theorem 2 and Section III B of [48]). Suppose the degree of the minimal polynomial of $G$ is $r$ at every point of the neighbourhood $U(p)$. Then, for arbitrary distinct $t_{1}, \ldots, t_{r+1} \in \mathbb{R}$ the functions $I_{t_{1}}, \ldots, I_{t_{r}}$ are functionally independent, and the function $I_{t_{r+1}}$ is a linear combination of the functions $I_{t_{1}}, \ldots, I_{t_{r}}$ (the coefficients of the linear combination are constant).

Recall that functions $f_{1}, \ldots, f_{r}$ are functionally independent, if their differentials are linearly independent almost everywhere.

In other words, the number of independent integrals among $I_{t}$ is the degree of the minimal polynomial. In particular, if the metrics are strictly nonproportional at a point $p$ (which immediately implies that they are strictly nonproportional at every point in a small neighbourhood $U(p)$ ), then the differentials of the integrals are linearly independent at almost every point of $T U(p)$.

\subsection{If geodesically equivalent metrics are strictly nonproportional at one point, then they are strictly nonproportional at almost every point.}

Let the geodesically equivalent metrics $g$ and $\bar{g}$ be strictly nonproportional at $p$. Consider a geodesic $\gamma$ passing through $p:=\gamma(0)$. Let us show that every point $q:=\gamma(\tau)$ of the geodesic has a neighborhood $U(q)$ such that at almost every point of the neighborhood the metrics are 
strictly nonproportional. Since every point can be reached by a finite sequence of geodesics, and since the condition "the minimal polynomial of $G$ has degree $n$ " is an open condition, this will prove the statement formulated in the title of this section.

As we recalled in Section 2.2, for almost every point of $T U(p)$ the differentials of the integrals $I_{t_{1}}, \ldots, I_{t_{n}}$ are linearly independent. Take a sequence of points $\left(p_{k}, v_{k}\right) \in T U(p)$ converging to $(\gamma(0), \dot{\gamma}(0))$ such that $d I_{t_{1}}\left(p_{k}, v_{k}\right), \ldots, d I_{t_{n}}\left(p_{k}, v_{k}\right)$ are linearly independent. Consider the sequence of the geodesics $\gamma_{k}$ such that $\gamma_{k}(0)=p_{k}$ and $\dot{\gamma}_{k}(0)=v_{k}$. For sufficiently large $k$, the geodesics exist up to time $\tau$ and the sequence $\left(\gamma_{k}(\tau), \dot{\gamma}_{k}(\tau)\right)$ converges to $(\gamma(\tau), \dot{\gamma}(\tau))$.

Since the integrals are preserved by the geodesic flow, the differentials of the integrals are also preserved by the geodesic flow. Hence, at the points $\left(\gamma_{k}(\tau), \dot{\gamma}_{k}(\tau)\right)$ the differentials of $I_{t_{1}}, \ldots, I_{t_{n}}$ are linearly independent. By Theorem 5, in an arbitrary small neighborhood of $\gamma_{k}(\tau)$ there exists a point $q_{k}$ such that the metrics are strictly nonproportional at $q_{k}$ implying the claim,

\subsection{If $g_{i k}, \bar{g}_{k j}$, and $\hat{g}_{k j}$ are linearly dependent at every point of a neighborhood $U$, then they are linearly dependent at every point of $M$.}

Within this section we assume that $g, \bar{g}, \hat{g}$ are geodesically equivalent metrics on a connected manifold $M$ of dimension $n \geq 3$. We consider the tensor $a_{i j}$ and the function $\lambda$ given by (3) and (4). The same objects for the pair of metrics $g, \hat{g}$ will be denoted by the capital letters $A$ and $\Lambda$, i.e.,

$$
A_{i j}:=\left|\frac{\operatorname{det}(\hat{g})}{\operatorname{det}(g)}\right|^{\frac{1}{n+1}} \cdot g_{i \alpha} \hat{g}^{\alpha \beta} g_{j \beta}, \quad \Lambda:=\frac{1}{2} A_{\alpha \beta} g^{\alpha \beta}
$$

We will first prove the following statement (essentially due to Weyl [52]).

Lemma 1. Suppose $a$ and $A$ are solutions of (5). Assume $a=C \cdot A$, where $C$ is a function. Then, $C$ is a constant.

Proof. Our proof is different from the proof of Weyl and is based on the ideas developed in [50]. Note that in the proof we use only the fact that the dimension is greater than one, i.e., it works in dimension 2 as well.

Consider two integrals $I_{0}$ of the form (7) constructed from $a$ (we keep the notation $I_{0}$ for it) and from $\hat{g}$ (we denote it by $\mathcal{I}_{0}$ ). If $a=C \cdot A$, then the integrals $I_{0}$ and $\mathcal{I}_{0}$ are proportional as well, direct calculations show that $I_{0}(\xi)= \pm C^{n-1} \cdot \mathcal{I}_{0}(\xi)$. Since the functions $I_{0}$ and $\mathcal{I}_{0}$ are constant along every trajectory of the geodesic flow, the coefficient of proportionality of these functions is also constant along every trajectory of the geodesic flow implying that it is constant everywhere. 
Now let us assume that at every point of the neighborhood $U$ the tensors $g, \bar{g}$, and $\hat{g}$ are linearly dependent. Then, for certain functions $c, d$ the tensors $g, a, A$ satisfy (probably in a smaller neighbourhood $U^{\prime} \subseteq U$; without loss of generality we can think that $U^{\prime}$ coincides with $U$.)

$$
a_{i j}=c g_{i j}+d A_{i j} .
$$

We will show that the functions $c, d$ are actually constants.

Differentiating (9) and substituting (5) and its analogue for the solution $A$, we obtain

$$
\lambda_{, i} g_{j k}+\lambda_{, j} g_{i k}=c_{, k} g_{i j}+d \Lambda_{, i} g_{j k}+d \Lambda_{, j} g_{i k}+d_{, k} A_{i j},
$$

which is evidently equivalent to

$$
\tau_{i} g_{j k}+\tau_{j} g_{i k}=c_{, k} g_{i j}+d_{, k} A_{i j},
$$

where $\tau_{i}=\lambda_{, i}-d \Lambda_{, i}$. We see that for every fixed $k$ the left-hand side of (11) is a symmetric matrix of the form $\tau_{i} v_{j}+\tau_{j} v_{i}$. If $c_{, k}$ is not proportional to $d_{, k}$, this will imply that $g_{i j}$ also is of the form $\tau_{i} v_{j}+\tau_{j} v_{i}$, which contradicts the non-degeneracy of $g$. If $c_{, k}=f \cdot d_{, k}$, then the coefficient $f$ of the proportionality should be a constant implying $d=$ const $\cdot c$ and $a_{i j}=c\left(g_{i j}+\right.$ const $\left.\cdot A_{i j}\right)$. Since the equation (5) is linear and since $g_{i j}$ and $A_{i j}$ are solutions of (5), their sum $g_{i j}+$ const $\cdot A_{i j}$ is also solution of (5). Then, Lemma 1 implies that $c$ is constant. Thus, $c$ and $d$ are constant in a neighbourhood $U$. Since the equation (5) is linear and of finite type, see [10], linear dependence of solutions in a neighbourhood implies linear dependence of the solutions everywhere.

Remark 5. Though we used that the dimension of the manifold is at least three, the statement is true in dimension two as well provided the Gauss curvature of $g$ is not constant, see [18].

\subsection{Main step of the proof of Theorem 1}

As we explained in Section 2.1, in view of Sections 2.3, 2.4, it is sufficient to prove Theorem 1 locally.

Assume the metrics $g, \bar{g}$, and $\hat{g}$ to be linearly independent at a point $p$. Assume that the metrics $g, \bar{g}$ are strictly nonproportional at $p$. Then, for some neighborhood $U(p)$ the metrics $g, \bar{g}$, and $\hat{g}$ are linearly independent, and the metrics $g, \bar{g}$ are strictly nonproportional at every point of $U(p)$.

It follows that the solution $a$ corresponding to $\bar{g}$, the solution $A$ corresponding to $\hat{g}$, and the metric $g$ are linearly independent at every point of $U(p)$, and the minimal polynomial of the $(1,1)$-tensor $a_{j}^{i}$ has degree $n$.

As in Section 2.3 we denote by $\Lambda$ the function (4) corresponding to $A$.

Let us first consider the case when $\lambda$ is constant. In this case, the equation (5) implies that $a_{i j}$ is covariantly constant. Since $a_{j}^{i}$ is self-adjoint with respect to $g$, there exists at every point a basis 
$\left(b_{1}(p), \ldots, b_{n}(p)\right)$ such that the matrix of $g$ is diagonal with \pm 1 on the diagonal and the matrix of $a_{j}^{i}$ is a Jordan-matrix. This basis is unique up to signs of the vectors, so locally $b_{i}$ can be taken to be smooth vector fields on the manifold. Since the vectors of the basis are invariantly constructed by two covariantly constant objects, they are covariantly constant as well. Hence, the metric $g$ is flat implying that $\bar{g}$ and $\hat{g}$ have constant sectional curvature.

In what follows we assume that $\lambda$ is not constant.

Theorem 1 is an easy consequence of the following two lemmas.

Lemma 2. Assume that $a$ and $A$ are solutions of (5) such that $a, A, g$ are linearly independent. Then, at every point there exists $K \in \mathbb{R}$ such that the tensor $Z_{i j k l}:=R_{i j, k l}-K \cdot\left(g_{i l} g_{j k}-g_{i k} g_{j l}\right)$ satisfies the condition

$$
a_{i}^{\alpha} Z_{\alpha j k l}+a_{j}^{\alpha} Z_{\alpha i k l}=0
$$

We see that by construction the tensor $Z_{i j k l}$ is skew-symmetric with respect to the first two indices. Note that in Lemma 2 we did not assume that the minimal polynomial of $a$ has degree $n$, i.e., the lemma is valid in a slightly more general setting than we need it.

Remark 6 . The tensor $Z$ plays an important role in the theory of geodesically equivalent metrics; it appears quite naturally in the investigation of special metrics such as Einstein metrics, pseudosymmetric metrics, Kähler metrics, see for example [14, 15] for details.

The next lemma shows that if in addition the minimal polynomial of $a$ has degree $n$, then condition (12) implies $Z_{i j k l}=0$, that is, the curvature of $g$ is constant. By Beltrami's Theorem (see for example [33], or the original papers [5] and [42]), the metrics $\bar{g}$ and $\hat{g}$ have constant sectional curvature as well which proves Theorem 1.

We will not use the indices $k$ and $l$ in the proof, so Lemma 3 is the matrix reformulation of the condition $a_{i}^{\alpha} Z_{\alpha j}+a_{j}^{\alpha} Z_{\alpha i}=0$ for an arbitrary $(1,1)$-tensor $a$ and a skew-symmetric $(0,2)$-tensor $Z$.

Lemma 3. Let $Z$, a be $n \times n$-matrices such that $Z$ is skew-symmetric. Assume the minimal polynomial of a has degree $n$. If $Z a+a^{t} Z=0$, then $Z=0$.

The proof of Lemma 3 is an easy exercise in linear algebra and is left to the reader (it is the kind of problem which is easier to prove than to understand the proof.) We recommend taking the coordinates such that the matrix $a$ is in Jordan form. One sees immediately by direct calculation that if the matrix $Z a$ is skew-symmetric, then $Z=0$.

\subsection{Proof of Lemma 2.}

The proof is by straightforward tensor calculations. The geometry behind the calculation could be understood with the help of [10]. There, the equations (5) were written in projectively invariant 
form (so that the equations for $g$ and for the geodesically equivalent metric $\bar{g}$ are the same). The prolongation of the equations was written as a connection on the projective tractor bundle, and the curvature of the connection was calculated. Its first part related to (the analogue of the tensor) $a$ is a trace-free object. Moreover, for most objects, the trace-free part of the covariant derivative coincides with the trace-free part of the corresponding derivative on the tractor bundle.

We will see that in the proof we consider the integrability conditions for the equations (5), and then "artificially" write all objects in trace-free form. At the end we obtain the required equation (12).

Note that in the proof we will essentially use the symmetries of the Riemannian curvature tensor, which have sense only if the affine connection is the Levi-Civita connection of a metric. That means, it is important for us that among the solutions of the projective-invariant analogue of (5) there is a non-degenerate solution.

In Section 3, we give another proof of Lemma 2 which is based on some ideas from the theory of integrable Hamiltonian systems on Lie algebras.

Proof of Lemma 2. Integrability conditions for the equation (5) are (we use the standard fact that $a_{i j, k l}-a_{i j, l k}=a_{i \alpha} R_{j k l}^{\alpha}+a_{\alpha j} R_{i k l}^{\alpha}$ for any $(0,2)$-tensor $\left.a_{i j}\right)$

$$
a_{i \alpha} R_{j k l}^{\alpha}+a_{\alpha j} R_{i k l}^{\alpha}=\lambda_{, l i} g_{j k}+\lambda_{, l j} g_{i k}-\lambda_{, k i} g_{j l}-\lambda_{, k j} g_{i l} .
$$

The same is true for the other solution $A$

$$
A_{i \alpha} R_{j k l}^{\alpha}+A_{\alpha j} R_{i k l}^{\alpha}=\Lambda_{, l i} g_{j k}+\Lambda_{, l j} g_{i k}-\Lambda_{, k i} g_{j l}-\Lambda_{, k j} g_{i l} .
$$

Starting from this point, the proof is purely algebraic: the statement of Lemma 2 is an algebraic corollary of (13) and (14). We emphasize this once again in the next section by giving another version of the proof in Lie-algebraic language.

Now let us multiply the equation (13) by $A_{s}^{l}$ and sum over $l$. After renaming indices, we obtain

$$
a_{i \alpha} R_{j k \beta}^{\alpha} A_{l}^{\beta}+a_{\alpha j} R_{i k \beta}^{\alpha} A_{l}^{\beta}=\lambda_{, \alpha i} A_{l}^{\alpha} g_{j k}+\lambda_{, \alpha j} A_{l}^{\alpha} g_{i k}-\lambda_{, k i} A_{j l}-\lambda_{, k j} A_{i l} .
$$

Using the symmetry of the Riemann tensor we obtain $a_{\alpha j} R_{i k \beta}^{\alpha}=a_{i}^{\alpha} R_{\alpha j, k \beta} A_{l}^{\beta}=a_{i}^{\alpha} R_{\beta k, j \alpha} A_{l}^{\beta}=$ $a_{i}^{\alpha} A_{\beta l} R_{k j \alpha}^{\beta}$. Substituting this in (15), we get

$$
a_{i}^{\alpha} A_{\beta l} R_{k i \alpha}^{\beta}+a_{j}^{\alpha} A_{\beta l} R_{k j \alpha}^{\beta}=\lambda_{, \alpha i} A_{l}^{\alpha} g_{j k}+\lambda_{, \alpha j} A_{l}^{\alpha} g_{i k}-\lambda_{, k i} A_{j l}-\lambda_{, k j} A_{i l} .
$$

Let us now symmetrise (16) with respect to $l$ and $k$ to obtain

$$
\begin{aligned}
& a_{i}^{\alpha}\left(A_{\beta l} R_{k j \alpha}^{\beta}+A_{\beta k} R_{l j \alpha}^{\beta}\right)+a_{j}^{\alpha}\left(A_{\beta k} R_{l i \alpha}^{\beta}+A_{\beta l} R_{k i \alpha}^{\beta}\right) \\
& =\lambda_{, \alpha i} A_{l}^{\alpha} g_{j k}+\lambda_{, \alpha j} A_{l}^{\alpha} g_{i k}-\lambda_{, k i} A_{j l}-\lambda_{, k j} A_{i l}+\lambda_{, \alpha i} A_{k}^{\alpha} g_{j l}+\lambda_{, \alpha j} A_{k}^{\alpha} g_{i l}-\lambda_{, l i} A_{j k}-\lambda_{, l j} A_{i k} .
\end{aligned}
$$


We see that the components in the brackets are the same as the left-hand side of the equation (14) with other indices. Substituting (14) into the first term of the left-hand side of (17), we obtain

$$
\begin{aligned}
a_{i}^{\alpha}\left(A_{\beta l} R_{k j \alpha}^{\beta}+A_{\beta k} R_{l j \alpha}^{\beta}\right) & =a_{i}^{\alpha}\left(\Lambda_{, \alpha l} g_{j k}+\Lambda_{, \alpha k} g_{j l}-\Lambda_{, j l} g_{k \alpha}-\Lambda_{, j k} g_{l \alpha}\right) \\
& =a_{i}^{\alpha} \Lambda_{, \alpha l} g_{j k}+a_{i}^{\alpha} \Lambda_{, \alpha k} g_{j l}-\Lambda_{, j l} a_{i k}-\Lambda_{, j k} a_{i l} .
\end{aligned}
$$

Similarly, the second term of the left-hand side of (17) is

$$
\begin{aligned}
a_{j}^{\alpha}\left(A_{\beta l} R_{k i \alpha}^{\beta}+A_{\beta k} R_{l i \alpha}^{\beta}\right) & =a_{j}^{\alpha}\left(\Lambda_{, \alpha l} g_{i k}+\Lambda_{, \alpha k} g_{i l}-\Lambda_{, i l} g_{k \alpha}-\Lambda_{, i k} g_{l \alpha}\right) \\
& =a_{j}^{\alpha} \Lambda_{, \alpha l} g_{u k}+a_{j}^{\alpha} \Lambda_{, \alpha k} g_{i l}-\Lambda_{, i l} a_{j k}-\Lambda_{, i k} a_{j l}
\end{aligned}
$$

Substituting $(18,19)$ in $(17)$, we obtain

$$
\begin{aligned}
& a_{i}^{\alpha} \Lambda_{, \alpha l} g_{j k}+a_{i}^{\alpha} \Lambda_{, \alpha k} g_{j l}-\Lambda_{, j l} a_{i k}-\Lambda_{, j k} a_{i l}+a_{j}^{\alpha} \Lambda_{, \alpha l} g_{i k}+a_{j}^{\alpha} \Lambda_{, \alpha k} g_{i l}-\Lambda_{, i l} a_{j k}-\Lambda_{, i k} a_{j l} \\
& =\lambda_{, \alpha i} A_{l}^{\alpha} g_{j k}+\lambda_{, \alpha j} A_{l}^{\alpha} g_{i k}-\lambda_{, k i} A_{j l}-\lambda_{, k j} A_{i l}+\lambda_{, \alpha i} A_{k}^{\alpha} g_{j l}+\lambda_{, \alpha j} A_{k}^{\alpha} g_{i l}-\lambda_{, l i} A_{j k}-\lambda_{, l j} A_{i k} .
\end{aligned}
$$

Collecting the terms involving $g$, we see that (20) can be written as

$$
\begin{aligned}
& \left(a_{i}^{\alpha} \Lambda_{, \alpha l}-\lambda_{, \alpha i} A_{l}^{\alpha}\right) g_{j k}+\left(a_{i}^{\alpha} \Lambda_{, \alpha k}-\lambda_{, \alpha i} A_{k}^{\alpha}\right) g_{j l}+\left(a_{j}^{\alpha} \Lambda_{, \alpha l}-\lambda_{, \alpha j} A_{l}^{\alpha}\right) g_{i k}+\left(a_{j}^{\alpha} \Lambda_{, \alpha k}-\lambda_{, \alpha j} A_{k}^{\alpha}\right) g_{i l} \\
& =\Lambda_{, j l} a_{i k}+\Lambda_{, j k} a_{i l}+\Lambda_{, i l} a_{j k}+\Lambda_{, i k} a_{j l}-\lambda_{, k i} A_{j l}-\lambda_{, k j} A_{i l}-\lambda_{, l i} A_{j k}-\lambda_{, l j} A_{i k} .
\end{aligned}
$$

We denote $\tau_{i l}:=a_{i}^{\alpha} \Lambda_{, \alpha l}-A_{l}^{\alpha} \lambda_{, \alpha i}$. In this notation, the equation (21) is

$$
\begin{aligned}
& \tau_{i l} g_{j k}+\tau_{i k} g_{j l}+\tau_{j l} g_{i k}+\tau_{j k} g_{i l} \\
= & \Lambda_{, j l} a_{i k}+\Lambda_{, j k} a_{i l}+\Lambda_{, i l} a_{j k}+\Lambda_{, i k} a_{j l}-\lambda_{, k i} A_{j l}-\lambda_{, k j} A_{i l}-\lambda_{, l i} A_{j k}-\lambda_{, l j} A_{i k} .
\end{aligned}
$$

Let us show that $\tau$ is symmetric. Multiplying with $g^{j k}$ and contracting with respect to $j, k$, we obtain

$$
\begin{aligned}
(n+2) \tau_{i l}+\left(\tau_{j k} g^{j k}\right) g_{i l}= & \Lambda_{, \alpha l} a_{i}^{\alpha}+\left(g^{j k} \Lambda_{, j k}\right) a_{i l}+\Lambda_{, i l}\left(a_{j k} g^{j k}\right)+\Lambda_{, i \alpha} a_{l}^{\alpha} \\
& -\lambda_{, \alpha i} A_{l}^{\alpha}-\left(\lambda_{, k j} g^{k j}\right) A_{i l}-\lambda_{, l i}\left(A_{j k} g^{j k}\right)-\lambda_{, l \alpha} A_{i}^{\alpha}
\end{aligned}
$$

We see that the right-hand side is symmetric with respect to $i$ and $l$. Then, so should be the left-hand-side implying $\tau_{i l}=\tau_{l i}$. We also see that the sum of the first, fourth, fifth and last terms of the right-hand side is $\tau_{i l}+\tau_{l i}=2 \tau_{i l}$. Then, the equation (23) is equivalent to

$$
\tau_{i l}=\frac{1}{n}\left(-\left(\tau_{j k} g^{j k}\right) g_{i l}+\left(g^{j k} \Lambda_{, j k}\right) a_{i l}+\Lambda_{, i l}\left(a_{j k} g^{j k}\right)-\left(\lambda_{, k j} g^{k j}\right) A_{i l}-\lambda_{, l i}\left(A_{j k} g^{j k}\right)\right) .
$$

Now we return to the equation (22). We alternate the equation with respect to $j$ and $k$ :

$$
\begin{aligned}
& \tau_{i k} g_{j l}+\tau_{j l} g_{i k}-\tau_{i j} g_{k l}-\tau_{k l} g_{i j} \\
= & \Lambda_{, j l} a_{i k}+\Lambda_{, i k} a_{j l}-\lambda_{, k i} A_{j l}-\lambda_{, l j} A_{i k}-\Lambda_{, k l} a_{i j}-\Lambda_{, i j} a_{k l}+\lambda_{, j i} A_{k l}+\lambda_{, l k} A_{i j} .
\end{aligned}
$$


Let us now rename $i \leftrightarrow k$ in $(25)$. We obtain

$$
\begin{aligned}
& \tau_{i k} g_{j l}+\tau_{j l} g_{i k}-\tau_{k j} g_{i l}-\tau_{i l} g_{k j} \\
= & \Lambda_{, j l} a_{i k}+\Lambda_{, i k} a_{j l}-\lambda_{, k i} A_{j l}-\lambda_{, l j} A_{i k}-\Lambda_{, i l} a_{k j}-\Lambda_{, k j} a_{i l}+\lambda_{, j k} A_{i l}+\lambda_{, l i} A_{k j} .
\end{aligned}
$$

Adding (22) with (26) and dividing by 2 for cosmetic reasons, we obtain

$$
\tau_{i k} g_{j l}+\tau_{j l} g_{i k}=\Lambda_{, j l} a_{i k}+\Lambda_{, i k} a_{j l}-\lambda_{, k i} A_{j l}-\lambda_{, l j} A_{i k}
$$

Substituting the expression for $\tau$ from (24), we have

$$
\begin{aligned}
& \frac{1}{n}\left(-\left(\tau_{\beta \alpha} g^{\beta \alpha}\right) g_{i k}+\left(g^{\alpha \beta} \Lambda_{, \alpha \beta}\right) a_{i k}+\Lambda_{, i k}\left(a_{\alpha \beta} g^{\alpha \beta}\right)-\left(\lambda_{, \alpha \beta} g^{\alpha \beta}\right) A_{i k}-\lambda_{, k i}\left(A_{\alpha \beta} g^{\alpha \beta}\right)\right) g_{j l} \\
+ & \frac{1}{n}\left(-\left(\tau_{\alpha \beta} g^{\alpha \beta}\right) g_{j l}+\left(g^{\alpha \beta} \Lambda_{, \alpha \beta}\right) a_{j l}+\Lambda_{, j l}\left(a_{\alpha \beta} g^{\alpha \beta}\right)-\left(\lambda_{, \alpha \beta} g^{\alpha \beta}\right) A_{j l}-\lambda_{, l j}\left(A_{\alpha \beta} g^{\alpha \beta}\right)\right) g_{i k} \\
= & \Lambda_{, j l} a_{i k}+\Lambda_{, i k} a_{j l}-\lambda_{, k i} A_{j l}-\lambda_{, l j} A_{i k} .
\end{aligned}
$$

Denoting $\left(\tau_{\beta \alpha} g^{\beta \alpha}\right)$ by $\tau,\left(\lambda_{, \beta \alpha} g^{\beta \alpha}\right)$ by $\mu,\left(\Lambda_{, \beta \alpha} g^{\beta \alpha}\right)$ by $\mathcal{M}$, and using that $\left(a_{\alpha \beta} g^{\alpha \beta}\right)=2 \lambda$, and $\left(A_{\alpha \beta} g^{\alpha \beta}\right)=2 \Lambda$, we obtain

$$
\begin{aligned}
& \frac{1}{n}\left(-\tau g_{i k}+\mathcal{M} a_{i k}+2 \Lambda_{, i k} \lambda-\mu A_{i k}-2 \lambda_{, k i} \Lambda\right) g_{j l} \\
+ & \frac{1}{n}\left(-\tau g_{j l}+\mathcal{M} a_{j l}+2 \Lambda_{, j l} \lambda-\mu A_{j l}-2 \lambda_{, l j} \Lambda\right) g_{i k} \\
= & \Lambda_{, j l} a_{i k}+\Lambda_{, i k} a_{j l}-\lambda_{, k i} A_{j l}-\lambda_{, l j} A_{i k} .
\end{aligned}
$$

Combining the terms, we obtain

$$
=\left(\begin{array}{l}
\left(-\frac{\tau}{2} g_{i k}+2 \Lambda_{, i k} \lambda\right) \frac{g_{j l}}{n}-\left(\frac{\tau}{2} g_{i k}+\lambda_{, k i} \Lambda\right) \frac{g_{j l}}{n}+\left(-\frac{\tau}{2} g_{j l}-2 \lambda_{, l j} \Lambda\right) \frac{g_{i k}}{n}+\left(-\frac{\tau}{2} g_{j l}+2 \Lambda_{, j l} \lambda\right) \frac{g_{i k}}{n} \\
\left.-\frac{1}{n} \mathcal{M} g_{j l}+\Lambda_{, j l}\right) a_{i k}+\left(\Lambda_{, i k}-\frac{1}{n} \mathcal{M} g_{i k}\right) a_{j l}-\left(\lambda_{, k i}-\frac{1}{n} \mu g_{i k}\right) A_{j l}-\left(\lambda_{, l j}-\frac{1}{n} \mu g_{j l}\right) A_{i k} .
\end{array}\right.
$$

Now let us calculate $\tau$ : we multiply (24) by $g^{i l}$ and sum over $i$ and $l$. After dividing by 2 , we get

$$
\tau=\frac{2}{n}(\mathcal{M} \lambda-\mu \Lambda)
$$

Substituting this expression into (27), we have

$$
=\left\{\begin{array}{l}
\left(-\frac{\mathcal{M}}{n} g_{i k}+\Lambda_{, i k}\right) \frac{2 \lambda}{n} g_{j l}-\left(-\frac{\mu}{n} g_{i k}+\lambda_{, k i}\right) \frac{2 \Lambda}{n} g_{j l}+\left(\frac{\mu}{n} g_{j l}-\lambda_{, l j}\right) \frac{2 \Lambda}{n} g_{i k}+\left(-\frac{\mathcal{M}}{n} g_{j l}+\Lambda_{, j l}\right) \frac{2 \lambda}{n} g_{i k} \\
\left.-\frac{1}{n} \mathcal{M} g_{j l}+\Lambda_{, j l}\right) a_{i k}+\left(\Lambda_{, i k}-\frac{1}{n} \mathcal{M} g_{i k}\right) a_{j l}-\left(\lambda_{, k i}-\frac{1}{n} \mu g_{i k}\right) A_{j l}-\left(\lambda_{, l j}-\frac{1}{n} \mu g_{j l}\right) A_{i k},
\end{array}\right.
$$


which is equivalent to

$$
=\begin{aligned}
& \left(\Lambda_{, i k}-\frac{\mathcal{M}}{n} g_{i k}\right)\left(a_{j l}-\frac{2 \lambda}{n} g_{j l}\right)+\left(\Lambda_{, j l}-\frac{\mathcal{M}}{n} g_{j l}\right)\left(a_{i k}-\frac{2 \lambda}{n} g_{i k}\right) \\
& \left(\lambda_{, k i}-\frac{\mu}{n} g_{i k}\right)\left(A_{j l}-\frac{2 \Lambda}{n} g_{j l}\right)+\left(\lambda_{, l j}-\frac{\mu}{n} g_{j l}\right)\left(A_{i k}-\frac{2 \Lambda}{n} g_{i k}\right)
\end{aligned}
$$

Denoting $B_{i k}:=\left(\Lambda_{, i k}-\frac{\mathcal{M}}{n} g_{i k}\right), b_{i k}:=\left(\lambda_{, k i}-\frac{\mu}{n} g_{i k}\right), d_{j l}:=\left(a_{j l}-\frac{2 \lambda}{n} g_{j l}\right), D_{j l}:=\left(A_{i k}-\frac{2 \Lambda}{n} g_{i k}\right)$, we see that $(28)$ is equivalent to

$$
B_{i k} d_{j l}+B_{j l} d_{i k}=b_{i k} D_{j l}+b_{j l} D_{i k} .
$$

Now, it is easy to see that the condition $B_{\alpha} d_{\beta}+B_{\beta} d_{\alpha}=b_{\alpha} D_{\beta}+b_{\beta} D_{\alpha}$ for dimensions $\geq 3$ implies that $B$ is proportional to $b$ and $D$ to $d$, or that $B$ is proportional to $D$ and $b$ to $d$. We see that condition (29) is essentially the same as this latter condition with $\alpha$ and $\beta$ representing the multi-indices $i k$ and $j l$. Since $g, a$, and $A$ are linearly independent, $D$ can not be proportional to $d$. Thus, $b$ is proportional to $d$ which implies that $\lambda_{, i j}$ is a linear combination of $a_{i j}$ and $g_{i j}$. The coefficients of the linear combination are not important for us. Substituting

$$
\lambda_{, i j}=\rho \cdot g_{i j}+K \cdot a_{i j}
$$

in (13), we obtain

$$
a_{i \alpha} Z_{j k l}^{\alpha}+a_{\alpha j} Z_{i k l}^{\alpha}=0
$$

where $Z_{j k l}^{i}=R_{j k l}^{i}-K \cdot\left(\delta_{l}^{i} g_{j k}-\delta_{k}^{i} g_{j l}\right)$. Clearly, equation (30) is equivalent to equation (12).

We see that by construction the tensor $Z_{i j k l}$ is skew-symmetric with respect to the first two indices as required in Lemma 3 (actually, the tensor $Z$ has the same symmetries as the curvature tensor, i.e., for example, skew-symmetric with respect to the last two indices as well, but we will need only the first two indices). Thus, Lemma 2 and, therefore, Theorem 1 are proved.

\section{Fubini theorem and sectional operators on semisimple Lie algebras}

In this section we discuss an unexpected and remarkable relationship between geodesically equivalent metrics and some special operators on semisimple Lie algebras which appeared in the theory of integrable systems.

We start with a brief overview on (one special type of) integrable Euler equations on semisimple Lie algebras (see $[6,11,23,38,39,40]$ for details).

Let $\mathfrak{g}$ be a semisimple Lie algebra, $R: \mathfrak{g} \rightarrow \mathfrak{g}$ an operator symmetric with respect to the Killing form $\langle$,$\rangle on \mathfrak{g}$. The differential equation

$$
\dot{x}=[R(x), x], \quad x \in \mathfrak{g},
$$


is Hamiltonian on $\mathfrak{g}$ with respect to the standard Lie-Poisson structure and called the Euler equation related to the Hamiltonian function $H(x)=\frac{1}{2}\langle R(x), x\rangle$.

A classical, interesting and extremely difficult problem is to find those operators $R: \mathfrak{g} \rightarrow \mathfrak{g}$ for which the system (31) is completely integrable.

One such operator was discovered by S. Manakov in [22] and his idea then led to an elegant general construction developed by A. Mischenko and A. Fomenko [38], called the argument shift method. In brief this construction can be presented as follows.

Assume that $R: \mathfrak{g} \rightarrow \mathfrak{g}$ satisfies the following identity

$$
[R(x), a]=[x, b], \quad x \in \mathfrak{g},
$$

for some $a, b \in \mathfrak{g}, a \neq 0$. Then the following statement holds

Theorem 6. [38] Let $R: \mathfrak{g} \rightarrow \mathfrak{g}$ be symmetric and satisfy (32). Then

1) the system (31) admits the following Lax representation with a parameter:

$$
\frac{d}{d t}(x+\lambda a)=[R(x)+\lambda b, x+\lambda a]
$$

2) the functions $f(x+\lambda a)$, where $f: \mathfrak{g} \rightarrow \mathbb{R}$ is an invariant of the adjoint representation, are first integrals of (31) for any $\lambda \in \mathbb{R}$ and, moreover, these integrals commute;

3) if $a \in \mathfrak{g}$ is regular, then (31) is completely integrable.

This construction has a very important particular case. If the Lie algebra $\mathfrak{g}$ admits a $\mathbb{Z}_{2}$-grading, i.e., a decomposition $\mathfrak{g}=\mathfrak{h}+\mathfrak{v}$ (direct sum of subspaces) such that $[\mathfrak{h}, \mathfrak{h}] \subset \mathfrak{h},[\mathfrak{h}, \mathfrak{v}] \subset \mathfrak{v},[\mathfrak{v}, \mathfrak{v}] \subset \mathfrak{h}$, then we may consider $R: \mathfrak{h} \rightarrow \mathfrak{h}$ satisfying (32) with $a, b \in \mathfrak{v}$, and Theorem 6 still holds if we replace $\mathfrak{g}$ by $\mathfrak{h}$.

The most important example for applications (in particular, in the theory of integrable tops) is $\mathfrak{g}=s l(n, \mathbb{R}), \mathfrak{h}=s o(n, \mathbb{R})$, with $a$ and $b$ symmetric matrices. This is the situation that was studied in the pioneering work by S. Manakov [22] leading to integrability of the Euler equations of $n$-dimensional rigid body dynamics.

From the algebraic point of view, the above construction still makes sense if we replace $s o(n)$ by $s o(p, q)$ and assume $a, b$ to be symmetric operators with respect to the corresponding indefinite form $g$. Moreover, if we complexify our considerations we do not even notice any difference. However, to indicate the presence (but not influence) of the bilinear form $g$, we shall denote the space of $g$-symmetric operators by $\operatorname{Sym}(g)$, and the Lie algebra of $g$-skew-symmetric operators by $s o(g)$. 
Definition 1 . We shall say that $R: s o(g) \rightarrow s o(g)$ is a sectional operator associated with $a, b \in$ $\operatorname{Sym}(g)$, if $R$ is symmetric with respect to the Killing form and the following identity holds:

$$
[R(x), a]=[x, b], \quad \text { for all } x \in s o(g) .
$$

We follow the terminology introduced by Fomenko and Trofimov in $[11,51]$ where they studied various generalizations of such operators. Strictly speaking, the above definition is just a particular case of a more general construction. The term "sectional" was motivated by the following reason. The identities (32) and (33) suggest that one may represent $R \operatorname{as~ad}_{a}^{-1} \mathrm{ad}_{b}$, but in general we cannot do so because $\mathrm{ad}_{a}$, as a rule, is not invertible. That is why the operator $R$ splits into different parts each of which acts independently on its own subspace (section).

A surprising relationship between sectional operators and geodesically equivalent metrics is explained by the following observation. Notice, first of all, that due to its algebraic symmetries (skew-symmetry with respect to $i, j$ and $k, l$ and symmetry with respect to permutation of pairs $(i j)$ and $(k l)$ ), the Riemann curvature tensor $R_{i j, k l}$ can be naturally considered as a symmetric operator $R: s o(g) \rightarrow s o(g)$ (strictly speaking we need to raise indices $i$ and $k$ by means of $g$ to get the tensor of the form $R_{j l}^{i k}$ ). Having this interpretation of $R$ in mind, we immediately obtain

Theorem 7. Let $g$ and $\bar{g}$ be geodesically equivalent nonproportional metrics, then the Riemann curvature tensor $R_{j l}^{i}{ }_{l}^{k}$ of the metric $g$ is a sectional operator in the sense of Definition 1 . More precisely,

$$
[R(x), a]=[x, b]
$$

where $a$ is the g-symmetric operator associated with the form $a_{i j}$ defined by (3), and $b$ is the g-symmetric operator associated with the form $2 \lambda_{, i j}$ (Hessian of $\operatorname{tr} a$ ).

The proof of this statement is just the observation that (33) is a translation of the compatibility condition (13) into Lie-algebraic language.

Before discussing the proof of the Fubini theorem in this "new" language, we make some remarks which could also be useful.

Notice, first of all, that in our new notation the condition "curvature is constant" (at a point) simply means that $R: s o(g) \rightarrow s o(g)$ is a scalar operator, i.e. $R(x)=K \cdot x$.

Furthermore, it is a very simple fact that (33) implies that $a$ and $b$ commute. Indeed, $\langle[b, a], x\rangle=$ $\langle a,[x, b]\rangle=\langle a,[R(x), a]\rangle=\langle[a, a], R(x)\rangle=0$ for any $x \in s o(g)$, so $[a, b]=0$. In the theory of projectively equivalent metrics this means that the operator $a$ commutes with the Hessian of its trace $2 \lambda=\operatorname{tr} a$. This fact is, of course, well known (and can be found for example in [44]), but the above proof seems to be the simplest one. Moreover, if instead of $a$ we substitute any element $\xi$ from its centralizer $C(a)$, we obviously get the same conclusion $[b, \xi]=0$, i.e., $b$ lies in the center of the centralizer of $a$. This means, in fact, that $b$ is a polynomial in $a$.

Finally, if $a$ is regular in the Lie-algebraic sense, i.e. its minimal polynomial coincides with the characteristic one, then the operator $\operatorname{ad}_{a}: \operatorname{so}(g) \rightarrow \operatorname{Sym}(g)$ has trivial kernel so that the 
sectional operator $R$ (i.e., the curvature tensor!) can be reconstructed from $a$ and $b$. Namely, $R(x)=\operatorname{ad}_{a}^{-1} \operatorname{ad}_{b}(x)$, a well-known formula in the theory of integrable systems on Lie algebras. If we take into account the fact that $b=P(a)=\lambda_{n-1} a^{n-1}+\lambda_{n-2} a^{n-2}+\cdots+\lambda_{1} a+\lambda_{0}$ (polynomial in $a$ ), then this formula can be rewritten as

$$
R(x)=\left.\frac{d}{d t} P(a+t x)\right|_{t=0} .
$$

Indeed, $[P(a+t x), a+t x]=0$ implies

$$
0=\left.\frac{d}{d t}[P(a+t x), a+t x]\right|_{t=0}=\left[\left.\frac{d}{d t} P(a+t x)\right|_{t=0}, a\right]+[P(a), x]
$$

i.e., $\left[\left.\frac{d}{d t} P(a+t x)\right|_{t=0}, a\right]=[x, b]$. Since $a$ is regular, we have $(34)$.

This shows, in particular, that the algebraic structure of the curvature tensor can be understood in terms of the operator $a$ only.

Using this language we now give another proof of the tensor part of the Fubini theorem (Lemmas 2 and 3$)$.

Assume that we have three geodesically equivalent metrics $g, \bar{g}$, and $\hat{g}$. Then the Riemann curvature tensor $R$ of the metric $g$ satisfies at the same time two identities :

$$
[R(x), a]=[x, b] \quad \text { and } \quad[R(x), A]=[x, B]
$$

where $a_{j}^{k}=g^{k i} a_{i j}, A_{j}^{k}=g^{k i} A_{i j}, b_{j}^{k}=2 g^{k i} \lambda_{, i j}, B_{j}^{k}=2 g^{k i} \Lambda_{, i j}$ (cf. (13) and (14)).

From now on, we may forget about the geometrical meaning of $a, b, A, B$ and start thinking of them as just certain $g$-symmetric operators. In addition, without loss of generality we may assume all these operators to be trace free (as, of course, they should be in the semisimple Lie algebra $s l(n, \mathbb{R})$ which stands behind this construction). Moreover, we are allowed to complexify all the objects so that instead of $s o(g)$ and $\operatorname{Sym}(g)$ we may simply consider the spaces of symmetric and skew-symmetric complex matrices.

The reformulation of the (algebraic part of) Fubini theorem are the following analogues of Lemmas 2 and 3 respectively.

Lemma 4. Let $R: s o(g) \rightarrow s o(g)$ be symmetric and satisfy (35). If a and $A$ are not proportional, then $b$ is proportional to $a$ and, therefore, $[R(x)-K \cdot x, a]=0$ for some $K \in \mathbb{R}$.

Lemma 5. If a is regular, i.e., its minimal polynomial coincides with the characteristic one, then the identity $[R(x)-K \cdot x, a]=0$ implies $R=K \cdot \mathrm{id}$ (i.e., the curvature is constant).

Proof of Lemma 4. Let $y$ and $z$ be arbitrary $g$-symmetric matrices, then $[A, y],[a, z] \in s o(g)$ and we have:

$$
[R([A, y]), a]=[[A, y], b], \quad[R([a, z]), A]=[[a, z], B]
$$


Since $R$ is symmetric with respect to the Killing form $\langle$,$\rangle we have$

$$
\begin{gathered}
\langle[[A, y], b], z\rangle=\langle[R([A, y]), a], z\rangle=\langle R([A, y]),[a, z]\rangle=\langle[A, y], R([a, z])\rangle= \\
\langle y,[R([a, z]), A]\rangle=\langle y,[[a, z], B]\rangle=\langle[[B, y], a], z\rangle
\end{gathered}
$$

Since $z$ is an arbitrary symmetric matrix, we conclude that

$$
[[A, y], b]=[[B, y], a] .
$$

This relation is an analogue of (20). Similarly, $[[a, y], B]=[[b, y], A]$. Using the Jacobi identity, it is not hard to see that

$$
[b, A]=[a, B]
$$

Rewriting (36) as

$$
y(B a-A b)+(a B-b A) y=B y a+a y B-b y A-A y b
$$

and noticing that $[b, A]=[a, B]$ implies $B a-A b=a B-b A$, we get

$$
y T+T y=B y a+a y B-b y A-A y b
$$

where $T$ denotes $a B-b A$ (this is an analogue of $\tau$ from (22)).

This formula can be considered as a relation between two linear operators acting on the space of symmetric matrices (the argument of both operators is $y \in \operatorname{Sym}(g)$ ). To get some consequences from this identity, we take a "kind of trace". Recall that we consider $A, a, B, b, y, T$ as usual symmetric (complex) matrices.

Instead of $y$ we substitute the symmetric matrix of the form $e_{i} v^{\top}+v e_{i}^{\top}$, where $e_{i}$ and $v$ are vector-columns $\left(e_{1}, \ldots, e_{n}\right.$ is the standard (orthonormal) basis), then apply the result to $e_{i}$ and take the sum over $i$. Here is the result:

$$
\begin{gathered}
\left(e_{i} v^{\top}+v e_{i}^{\top}\right) T e_{i}+T\left(e_{i} v^{\top}+v e_{i}^{\top}\right) e_{i}=B\left(e_{i} v^{\top}+v e_{i}^{\top}\right) a e_{i}+\ldots \\
e_{i}\left(T v, e_{i}\right)+v\left(T e_{i}, e_{i}\right)+T e_{i}\left(v, e_{i}\right)+T v\left(e_{i}, e_{i}\right)=B e_{i}\left(a v, e_{i}\right)+B v\left(a e_{i}, e_{i}\right)+\ldots
\end{gathered}
$$

Using obvious facts from Linear Algebra such as

$$
\sum_{i}\left(T e_{i}, e_{i}\right)=\operatorname{tr} T, \quad \sum_{i}\left(e_{i}, e_{i}\right)=n, \quad \sum_{i} e_{i}\left(v, e_{i}\right)=v,
$$

we get

$$
T v+\operatorname{tr} T \cdot v+T v+n \cdot T v=B a v+\operatorname{tr} a \cdot B v+\ldots
$$

Taking into account that $a, A, b, B$ are all trace free we have

$$
((n+2) T+\operatorname{tr} T \cdot \mathrm{Id}) v=(B a+a B-A b-b A) v .
$$


Since $v$ is arbitrary and $T=B a-A b=a B-b A$, we finally get

$$
n T+\operatorname{tr} T \cdot \operatorname{Id}=0,
$$

but this simply means that $T=0$. Hence we come to the identity of the form

$$
B y a+a y B=b y A+A y b .
$$

It remains to use the following simple statement: if $a, b, A, B$ are symmetric, $a \neq 0$ and (37) holds for any symmetric $y$, then either $b=K \cdot a$, or $A=K \cdot a$ for some constant $K \in \mathbb{R}$.

By our assumption, $a$ and $A$ are not proportional, so we conclude that $b=K \cdot a$ and therefore the identity $[R(x), a]=[x, b]$ becomes $[R(x)-K \cdot x, a]=0$, as needed.

Notice that (37) and the rest of the proof almost literally repeat (29) and the end of the proof of Lemma 2,

Proof of Lemma 5. Let $[R(x)-K \cdot x, a]=0$ and $a$ be regular. It is a well known algebraic fact that the centralizer of a regular matrix $a$ is generated by the powers of $a$. In particular, the centralizer of $a$ consists of $g$-symmetric matrices. On the other hand, $R(x)-K \cdot x$ is skewsymmetric. Thus, $R(x)-K \cdot x$ has to be zero for any $x$, i.e., $R=K \cdot$ id, as was to be proved.

\section{References}

[1] A. V. Aminova, 'A Lie problem, projective groups of two-dimensional Riemann surfaces, and solitons', Izv. Vyssh. Uchebn. Zaved. Mat. 1990, no. 6, 3-10; translation in Soviet Math. (Iz. VUZ) 34 (1990), no. 6, 1-9.

[2] A. V. Aminova, 'Pseudo-Riemannian manifolds with general geodesics', Russian Math. Surveys 48 (1993), no. 2, 105-160.

[3] A. V. Aminova, 'Projective transformations of pseudo-Riemannian manifolds. Geometry, 9', J. Math. Sci. (N. Y.) 113 (2003), no. 3, 367-470.

[4] S. Benenti, 'Special symmetric two-tensors, equivalent dynamical systems, cofactor and bicofactor systems', Acta Appl. Math. 87 (2005), no. 1-3, 33-91.

[5] E. Beltrami, 'Resoluzione del problema: riportari i punti di una superficie sopra un piano in modo che le linee geodetische vengano rappresentante da linee rette', Ann. Mat., 1 (1865), no. $7,185-204$.

[6] O. I. Bogoyavlensky, 'Integrable Euler equations on Lie algebras, arising in problems of mathematical physics', Izv. Akad. Nauk SSSR Ser. Mat. 48 (1984), no. 5, 883-938 (Russian). 
[7] A. V. Bolsinov, V. S. Matveev, 'Geometrical interpretation of Benenti systems', J. Geom. Phys. 44 (2003), 489-506.

[8] R. L. Bryant, G. Manno, V. S. Matveev, 'A solution of a problem of Sophus Lie: Normal forms of 2-dim metrics admitting two projective vector fields', Math. Ann. 340 (2008), no. 2, 437-463, arXiv:0705.3592 .

[9] R. L. Bryant, M. Dunajski, M. Eastwood, 'Metrisability of two-dimensional projective structures', to appear in J. Diff. Geom. arXiv:0801.0300.

[10] M. Eastwood, V. S. Matveev, 'Metric connections in projective differential geometry', Symmetries and Overdetermined Systems of Partial Differential Equations (Minneapolis, MN, 2006), 339-351, IMA Vol. Math. Appl., 144 (2007), Springer, New York.

[11] A. T. Fomenko, V. V. Trofimov, Integrable systems on Lie Algebras and Symmetric Spaces (Gordon and Breach, London/New York, 1988).

[12] G. Fubini, 'Sui gruppi transformazioni geodetiche', Mem. Acc. Torino 53 (1903), 261-313.

[13] G. Fubini, 'Sulle coppie di varieta geodeticamente applicabili', Acc. Lincei 14 (1905), 678-683 (1 Sem.), 315-322 (2 Sem.).

[14] V. A. Kiosak, I. Mikesh, 'On the degree of mobility of Riemannian spaces with respect to geodesic mappings'. (Russian) The geometry of imbedded manifolds, 124 (Moskov. Gos. Ped. Inst., Moscow, 1986) 35-39.

[15] V. A. Kiosak and Ĭ. Mikesh, 'On geodesic mappings of Einstein spaces', Izv. Vyssh. Uchebn. Zaved. Mat. 2003, no. 11, 36-41; translation in Russian Math. (Iz. VUZ) 47 (2003), no. 11, $32-37$.

[16] G. Koenigs, 'Sur les géodesiques a intégrales quadratiques', Note II from Darboux' "Leçons sur la théorie générale des surfaces", Vol. IV, Chelsea Publishing, 1896.

[17] B. S. Kruglikov, V. S. Matveev, 'Vanishing of the entropy pseudonorm for certain integrable systems', Electron. Res. Announc. Amer. Math. Soc. 12 (2006), 19-28.

[18] B. Kruglikov, 'Invariant characterization of Liouville metrics and polynomial integrals', arXiv:0709.0423

[19] J.-L. Lagrange, 'Sur la construction des cartes géographiques', Novéaux Mémoires de l'Académie des Sciences et Bell-Lettres de Berlin, 1779.

[20] T. Levi-Civita, 'Sulle trasformazioni delle equazioni dinamiche', Ann. di Mat., serie $2^{a}, 24$ (1896), 255-300.

[21] S. Lie, 'Untersuchungen über geodätische Kurven', Math. Ann. 20 (1882); Sophus Lie Gesammelte Abhandlungen, Band 2, erster Teil, (Teubner, Leipzig, 1935) 267-374. 
[22] S. V. Manakov, 'Note on the integration of Euler's equation of the dynamics of an $N$ dimensional rigid body', Funct. Anal. Appl. 11 (1976), 328-329.

[23] J. E. Marsden, T. S. Ratiu, Introduction to Mechanics and Symmetry (Springer Verlag, New York, 1999).

[24] V. S. Matveev, P. J. Topalov, 'Trajectory equivalence and corresponding integrals', Regul. Chaot. Dyn. 3 (1998), no. 2, 30-45.

[25] V. S. Matveev and P. J. Topalov, 'Quantum integrability for the Beltrami-Laplace operator as geodesic equivalence', Math. Z. 238 (2001), 833-866.

[26] V. S. Matveev and P. J. Topalov, 'Integrability in theory of geodesically equivalent metrics', J. Phys. A., 34 (2001), 2415-2433.

[27] V. S. Matveev, 'Three-dimensional manifolds having metrics with the same geodesics', Topology 42 (2003) no. 6, 1371-1395.

[28] V. S. Matveev, Hyperbolic manifolds are geodesically rigid, Invent. Math. 151 (2003), 579609.

[29] V. S. Matveev, Die Vermutung von Obata für Dimension 2, Arch. Math. 82 (2004), 273-281.

[30] V. S. Matveev, Solodovnikov's theorem in dimension two, Dokl. Math. 69 (2004), no. 3, $338-341$.

[31] V. S. Matveev, 'Lichnerowicz-Obata conjecture in dimension two', Comm. Math. Helv. 81 (2005) no. 3, 541-570.

[32] V. S. Matveev, 'Beltrami problem, Lichnerowicz-Obata conjecture and applications of integrable systems in differential geometry', Trudy Sem. Vektor. Tenzor. Anal. 26 (2005), 214-238 (Russian).

[33] V. S. Matveev, 'Geometric explanation of Beltrami theorem', Int. J. Geom. Methods Mod. Phys. 3 (2006), no. 3, 623-629.

[34] V. S. Matveev, 'On degree of mobility of complete metrics', Adv. Stud. Pure Math. 43 (2006), 221-250.

[35] V. S. Matveev, 'Proof of projective Lichnerowicz-Obata conjecture', J. Diff. Geom. (2007), 75 (2007), 459-502.

[36] V. S. Matveev, 'A solution of another S. Lie Problem: 2-dim metrics admitting projective vector field', submitted to Math. Ann., arXiv:math/0802.2344

[37] J. Mikes, 'Geodesic mappings of affine-connected and Riemannian spaces. Geometry, 2', J. Math. Sci. 78 (1996), no. 3, 311-333. 
[38] A. S. Mishchenko, A. T. Fomenko, 'Euler equations on finite-dimensional Lie groups', Izv. Acad. Nauk SSSR, Ser. matem. 42 (1978) no. 2, 396-415 (Russian); English translation: Math. USSR-Izv. 12 (1978), no.2, 371-389.

[39] A. M. Perelomov, Integrable systems of classical mechanics and Lie algebras. Vol. I. (Birkäuser Verlag, Basel, 1990).

[40] A. G. Reyman, M. A. Semenov-Tian-Shansky, Integrable systems. A group theoretic approach. (Institute of Computer Science, Moscow-Izhevsk, 2003) (Russian).

[41] J. A. Schouten, 'Erlanger Programm und Übertragungslehre. Neue Gesichtspunkte zur Grundlegung der Geometrie', Rendiconti Palermo 50 (1926), 142-169.

[42] F. Schur, 'Ueber den Zusammenhang der Räume constanter Riemann'schen Krümmumgsmaasses mit den projektiven Räumen', Math. Ann. 27 (1886), 537-567.

[43] I. G. Shandra, 'On the geodesic mobility of Riemannian spaces', Math. Notes 68 (2000), no. $3-4,528-532$.

[44] N. S. Sinjukov, Geodesic mappings of Riemannian spaces (Nauka, Moscow, 1979), (in Russian) .

[45] A. S. Solodovnikov, 'Projective transformations of Riemannian spaces', Uspehi Mat. Nauk (N.S.) 11 (1956), no. 4 (70), 45-116 (Russian).

[46] A. S. Solodovnikov, 'Spaces with common geodesics', Trudy Sem. Vektor. Tenzor. Anal. 11 (1961), 43-102.

[47] A. S. Solodovnikov, 'Geometric description of all possible representations of a Riemannian metric in Levi-Civita form', Trudy Sem. Vektor. Tenzor. Anal. 12 (1963), 131-173.

[48] P. Topalov, 'Geodesic hierarchies and involutivity', J. Math. Phys. 42 (2001), no. 8, 38983914.

[49] P. Topalov, 'Commutative conservation laws for geodesic flows of metric admitting projective symmetry', Math. Res. Lett. 9 (2002), no. 1, 65-72.

[50] P. J. Topalov and V. S. Matveev, 'Geodesic equivalence via integrability', Geom. Dedicata 96 (2003), 91-115.

[51] V. V. Trofimov and A. T. Fomenko, 'Dynamical systems on the orbits of linear representations of Lie groups and the complete integrability of certain hydrodynamical systems', Funct. Anal. Appl. 17 (1983), 2-29.

[52] H. Weyl, 'Zur Infinitisimalgeometrie: Einordnung der projektiven und der konformen Auffasung', Nachrichten von der K. Gesellschaft der Wissenschaften zu Göttingen, MathematischPhysikalische Klasse, 1921; "Selecta Hermann Weyl", Birkhäuser Verlag, Basel und Stuttgart, 1956. 
[53] V. E. Zakharov, 'Description of the $n$-orthogonal curvilinear coordinate systems and Hamiltonian integrable systems of hydrodynamic type. I. Integration of the Lamé equations', Duke Math. J. 94 (1998), no. 1, 103-139.

[54] Z. Zakirova, 'On projective group properties of the 6D pseudo-Riemannian space', Czechoslovak J. Phys. 55 (2005), no. 11, 1541-1544.

\author{
Alexey V.Bolsinov, \\ School of Mathematics, Loughborough University, \\ Loughborough, LE11 3TU, UK \\ e-mail: A.Bolsinov@lboro.ac.uk, \\ Volodymyr Kiosak, \\ Institute of Physics and Mathematics, \\ K. Ushynsky South Ukraine Pedagogical University, \\ Odessa, Ukraine \\ e-mail: vkiosak@ukr.net \\ Vladimir S. Matveev \\ Institute of Mathematics, \\ Friedrich-Schiller-Universität Jena, \\ 07737 Jena Germany \\ e-mail: matveev@minet.uni-jena.de
}

